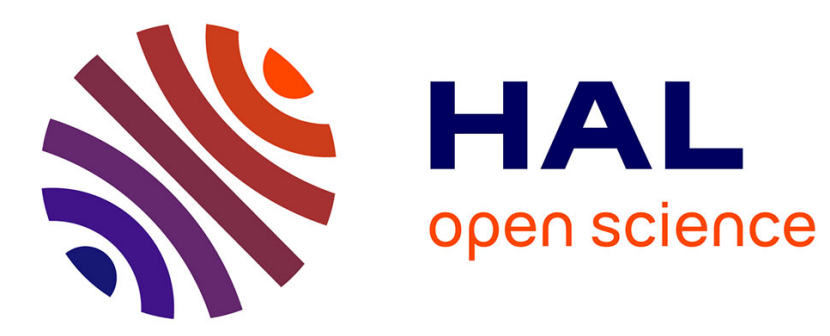

\title{
Online fuzzy temporal operators for complex system monitoring
}

Jean-Philippe Poli, Laurence Boudet, Bruno Espinosa, Laurence Cornez

\section{To cite this version:}

Jean-Philippe Poli, Laurence Boudet, Bruno Espinosa, Laurence Cornez. Online fuzzy temporal operators for complex system monitoring. ISIPTA '17 and ECSQARU 2017 - 14th European Conference on Symbolic and Quantitative Approaches to Reasoning and Uncertainty, Jul 2017, Lugano, Switzerland. pp.375-384, 10.1007/978-3-319-61581-3_34. cea-01809219

\section{HAL Id: cea-01809219 https://hal-cea.archives-ouvertes.fr/cea-01809219}

Submitted on 26 Feb 2022

HAL is a multi-disciplinary open access archive for the deposit and dissemination of scientific research documents, whether they are published or not. The documents may come from teaching and research institutions in France or abroad, or from public or private research centers.
L'archive ouverte pluridisciplinaire HAL, est destinée au dépôt et à la diffusion de documents scientifiques de niveau recherche, publiés ou non, émanant des établissements d'enseignement et de recherche français ou étrangers, des laboratoires publics ou privés. 


\title{
Online fuzzy temporal operators for complex system monitoring
}

\author{
Jean-Philippe Poli Laurence Boudet Bruno Espinosa \\ Laurence Cornez \\ CEA, LIST, Data Analysis and System Intelligence Laboratory, \\ 91191 Gif-sur-Yvette cedex, France. \\ firstname.lastname@cea.fr
}

February 25, 2022

\begin{abstract}
Online fuzzy expert systems can be used to process data and event streams, providing a powerful way to handle their uncertainty and their inaccuracy. Moreover, human experts can decide how to process the streams with rules close to natural language. However, to extract high level information from these streams, they need at least to describe the temporal relations between the data or the events.

In this paper, we propose temporal operators which relies on the mathematical definition of some base operators in order to characterize trends and drifts in complex systems. Formalizing temporal relations allows experts to simply describe the behaviors of a system which lead to a break down or an ineffective exploitation. We finally show an experiment of those operators on wind turbines monitoring.
\end{abstract}

\section{Introduction}

Complex systems are now equipped with hundreds of sensors which deliver continuous signals. Sensors provide either measurements at a dynamic or constant sampling rate (i.e. data streams, e.g. connected thermometers), either events whenever they are detected (i.e. event streams, e.g. presence detectors). Such streams are generally processed, filtered and combined to get higher level information. These operations can be applied to predictive maintenance of complex systems.

Predictive maintenance consists in monitoring an engineering system in order to detect changes in its exploitation and prevent damages. Having a continuous report of in-service systems allows an optimal use of it, the avoidance of important damages and early-stage failure detection. Moreover, it changes the organization of maintenance services by replacing scheduled and periodic maintenance and by minimizing the involvement of operators. 
Artificial intelligence plays an important role in predictive maintenance [4] and provides system-specific solutions : signal processing and statistical learning techniques have been successfully applied to obtain a type of damage or a type of risk. Predictive maintenance mainly relies on data from process sensors (temperature, pressure, etc.) and test sensors (vibration, acoustic, humidity, etc.) [3]. In order to better handle the sensors inaccuracy and the uncertainty in the assessment of the system's state, fuzzy logic has been applied to predictive maintenance $[8,12]$.

Our work consists in developing an online fuzzy expert system which can take data or event streams as input. The goal is to reinforce the expressivity of such systems to let experts author their own rules with complex fuzzy relations. Gathering the knowledge of different experts can be a suitable approach to predictive maintenance, avoiding some difficulties of the techniques described formerly:

- no past data are needed to build the models;

- the decision can be explained through the trace of activated rules.

In the case of predictive maintenance, the rules consist in detecting patterns in time-series which lead to a damage. Numerous authors $[1,2,11]$ state temporal relations are a prerequisite to describe such patterns. One can distinguish different approaches. On the one hand, fuzzy temporal relations $[2,5,10]$ can be used to describe the temporality of events but are not always relevant for online causal reasoning. On the other hand, some papers suggest to linguistically describe time-series $[6,7]$ using fuzzy natural logic, specifically on complete time-series, i.e. in an offline way.

In this article, we remind 3 base fuzzy temporal relations which are then combined into more complex relations. The compositional paradigm we use allows to create new intuitive relations because they combined simple operators. The new operators are the first of a series of temporal operators which can be used to describe time-series. In our work, we make the following assumptions:

- sensors give correct timestamps: there is no uncertainty in the acquisition timestamps, but we take into account the vagueness in the relations between the timestamps;

- sensors values are fuzzified to both manipulate linguistic terms and manage their inaccuracy.

The article is organized as follows: the next section presents the previous work and the notations. The new temporal operators are described in section 3. In section 4, we describe their use by an application to wind turbine predictive maintenance. Finally, section 5 draws the conclusions and perspectives of this work. 


\section{Previous work}

In our previous work, we introduced a compositional paradigm which consists in deriving specialized operators from base operators in the temporal domain [9]. In this paper, we take advantage from these operators to build new temporal ones for online characterization of time-series.

The temporal operators use two concepts to deal with event streams [9]. On the one hand, expiration is the faculty for a temporal expression to yell that its value has expired and must be re-evaluated. On the other hand, they are applied on a scope. A scope is a fuzzy set defined on a temporal domain, anchored at the present moment, and whose membership function gives the importance of a moment in this temporal domain. For instance, figure 1(c) shows such a scope representing "the last 10 seconds". Both concepts ensure a satisfying computational cost and allow an online execution.

Let $E$ be a fuzzy expression, $\operatorname{eval}(E, t)$ be the value of $E$ at time $t$. Let $S$ be a fuzzy scope and $\mu_{S}$ its membership function. In the remainder of this paper, we will use the following temporal operators:

- The occurrence operator which indicates if an expression has a degree of fulfillment strictly greater than 0 throughout the scope :

$$
\operatorname{Occ}\left(E, S, t_{\text {now }}\right)=\bigvee_{t \in \operatorname{supp}(S)} \operatorname{eval}(E, t) \wedge \mu_{S}(t)
$$

When its value is strictly greater than 0 , it means that at least at one moment of the scope, the operand expression has been observed. It is a disjunction over all the moments $t_{i}$ in the scope of conjunctions of the operand value at time $t_{i}$ and the value of the scope membership function for $t_{i}$.

- The ratio operator which aggregates the different degrees of fulfillment of the operand expression $E$ throughout a scope $S$ :

$$
\operatorname{Ratio}\left(E, S, t_{\text {now }}\right)=\frac{\int_{t \in \operatorname{supp}(S)} \operatorname{eval}(E, t) \wedge \mu_{S}(t)}{\int_{t \in \operatorname{supp}(S)} \mu_{S}(t)}
$$

It aggregates the different values of the operand $E$ on the scope $S$, divided by the area under the scope membership function. It is related to Zadeh's relative count applied on a fuzzy scope.

- The persistence operator which indicates if at each moment of $S$, the degree of fulfillment of $E$ is strictly greater than 0 :

$$
\operatorname{StrictPers}\left(E, S, t_{\text {now }}\right)=\neg \operatorname{Occ}\left(\neg E, S, t_{\text {now }}\right) .
$$

It equals 0 if there exists a moment $t_{i}$ in the scope $S$ such as eval $\left(E, t_{i}\right)=0$. This is why we called it "strict". To moderate its definition, we can either replace the Occ operator by the Ratio inside its definition, or simply use Ratio instead of StrictPers. 


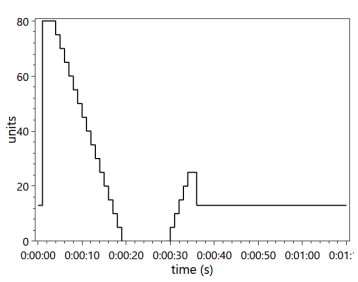

(a) Signal of Input1

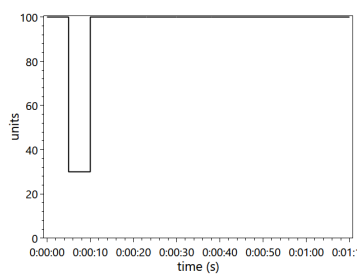

(b) Signal of Input2

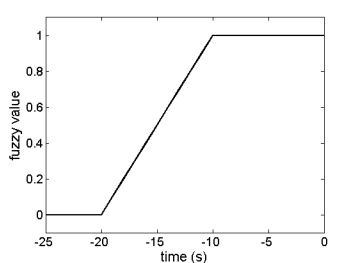

(c) Fuzzy scope for the last 10 seconds

Figure 1: Examples of two signals and a fuzzy scope

In the next section, we use these operators to define new temporal operators to both characterize trends of time-series and to compare two of them. Adopting an iterative approach, we first define the following operators and we will add new ones when are not sufficient anymore.

\section{Signal characterization operators}

To illustrate the behavior of the operators, we introduce some examples of input signals and parameters we will use throughout this section.

For the sake of comprehension, figures 1(a) and 1(b) show two simple simulated signals. We will use these signals to illustrate the behavior of the operators and in the section 4 , we will use more realistic signals.

In the remainder of this section, without loss of generality, the operators are defined upon the Ratio operator. As a consequence of the use of the Ratio operator, those operators are considered tolerant. Thus, if at some moment the input signal is changing for a short while, the direct effect of its change is smoothed. If a more strict behavior is needed, it is possible to replace the Ratio by the StrictPers operator.

\subsection{Growth, decline and variation}

In predictive maintenance, it is important to be able to characterize drifts of some sensors, because it can lead to the detection of a damage. The goal here is to monitor the growth or the decline of an input value with operators such as:

input $\langle a d v e r b\rangle$ decreases/increases throughout $S$.

where adverb is a fuzzy set which represents, for example, "slowly" or "significantly" and $S$ is a fuzzy scope.

To compute a degree of fulfillment for such relations, saving all the values in the scope is not necessary. We chose instead to compute the gradient between the two last samples and then to characterize its direction with a fuzzy set

corresponding to the adverb. The fuzzy set is thus defined on a quarter of 


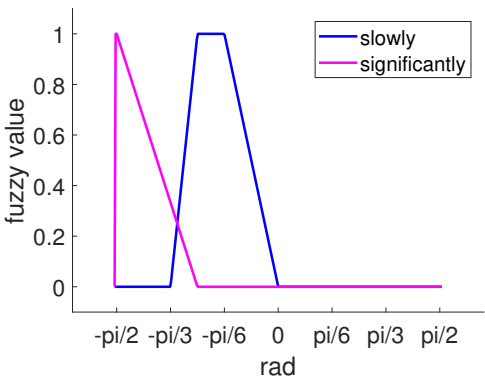

(a) Examples of "slowly" and "significantly" membership functions

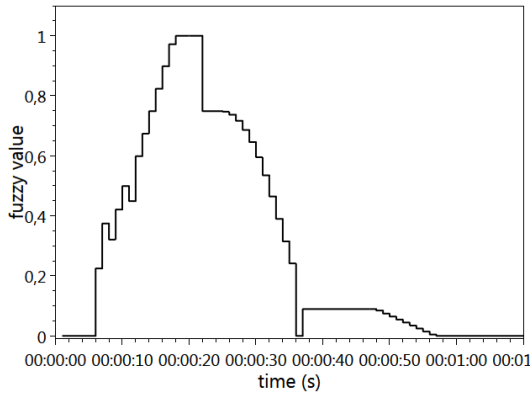

(b) "Input1 significantly decreases throughout the last 10 seconds"

Figure 2: Examples of membership functions for the adverbs of the Decreases operator and result on Input 1

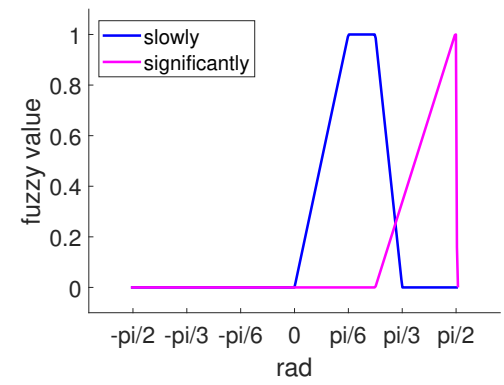

(a) Examples of "slowly" and "significantly" membership functions

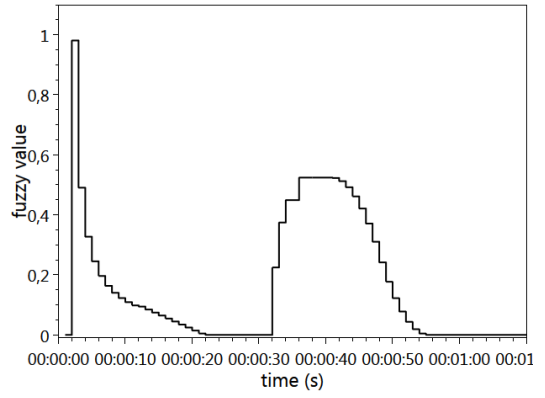

(b) "Input1 significantly increases throughout the last 10 seconds"

Figure 3: Examples of membership functions for the adverbs of the Increases operator and result on Input 1

the trigonometric circle (top-right quadrant for the growth and bottom-right quadrant for the decline). Figures 2(a) and 3(a) show an example of membership functions for adverbs "slowly" and "significantly" applied respectively to the decline and the growth operator.

To aggregate the characterizations of the gradient over the scope, we can use the Ratio. Thus, the Decreases operator can be defined as:

$$
\operatorname{Decreases}\left(I, S, \mu_{g}, t_{\text {now }}\right)=\operatorname{Ratio}\left(\mu_{g}\left(\operatorname{grad}\left(I, t_{\text {now }}\right)\right), S, t_{\text {now }}\right)
$$

where $I$ is the real input of the system whose values change, grad is the direction of the gradient, and $\mu_{g}$ is the membership function of the adverb fuzzy set.

The Increases operator only differs from the Decreases operator because of the definition domain and the membership function of the adverb fuzzy set. 


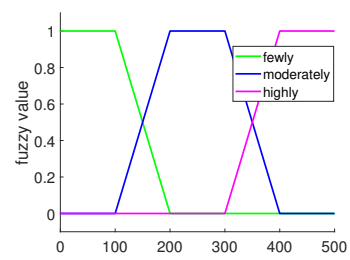

(a) Examples of "fewly", "moderately" and "highly" membership functions

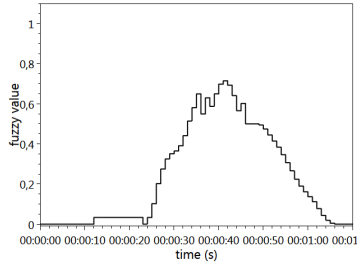

(b) "Input1 varies fewly throughout the last 10 seconds"

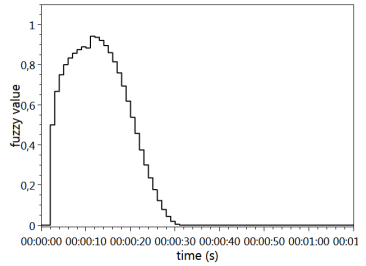

(c) "Input1 varies highly throughout the last 10 seconds"

Figure 4: Examples of membership functions for the adverbs of the Varies operator and results on Input 1

Figures 2(b) and 3(b) show respectively the result of operators Decreases and Increases on the first input whose signal is shown in figure 1(a).

In a similar way, it is useful to be able to tell that the value of an input remains stable over time, with an operator like:

$$
\text { input varies }\langle\text { adverb }\rangle \text { throughout } S
$$

where adverb is a fuzzy set which represents, for instance, "fewly" or "highly". The definition of the Varies operator is based on the variance of its signal over $S$ and on a fuzzy set which defines the adverb by characterizing the variance. The Varies operator is defined by:

$$
\operatorname{Varies}\left(I, S, \mu_{v}, t_{\text {now }}\right)=\operatorname{Ratio}\left(\mu_{v}(\operatorname{Var}(I, \operatorname{supp}(S))), S, t_{\text {now }}\right)
$$

where $I$ is an input of the system whose value changes, Var is the variance of the signal $I(t)$ over $S$ and $\mu_{v}$ is the membership function of the adverb fuzzy set.

Figures 4(b) and 4(c) show the results of the Varies operator on the first input (see figure 1(a)), using respectively the adverbs "fewly" and "highly" described in figure $4(\mathrm{a})$.

\subsection{Comparison}

The last family of operators in this article concerns comparison between two input values throughout a scope; one of them can be a fixed value, for instance a threshold. For instance, an expert may want to express that the signal of an input is extremely less than another value:

input1 is $\langle a d v e r b\rangle$ less/greater/close than/to input2 throughout $S$.

The idea behind these operators is to compare at each time the two values and to characterize the difference between them with a fuzzy set (the adverb). 
Then, we aggregate the point-to-point comparisons with the Ratio operator. Thus, we can define LessThan, GreaterThan, CloseTo as:

$$
\begin{aligned}
\operatorname{LessThan}\left(I_{1}, I_{2}, S, \mu_{l t}, t_{\text {now }}\right) & =\operatorname{Ratio}\left(\mu_{l t}\left(I_{1}\left(t_{\text {now }}\right)-I_{2}\left(t_{\text {now }}\right)\right), S, t_{\text {no }}((9))\right. \\
\operatorname{GreaterThan}\left(I_{1}, I_{2}, S, \mu_{\text {gt }}, t_{\text {now }}\right) & =\operatorname{Ratio}\left(\mu_{\text {gt }}\left(I_{1}\left(t_{\text {now }}\right)-I_{2}\left(t_{\text {now }}\right)\right), S, t_{\text {no }}(7)\right) \\
\operatorname{ClosteTo}\left(I_{1}, I_{2}, S, \mu_{c t}, t_{\text {now }}\right) & =\operatorname{Ratio}\left(\mu_{c t}\left(I_{1}\left(t_{\text {now }}\right)-I_{2}\left(t_{\text {now }}\right)\right), S, t_{\text {now }}(8)\right)
\end{aligned}
$$

where $\mu_{l t}, \mu_{g t}$ and $\mu_{c t}$ are the membership functions of the adverb fuzzy set which characterizes the difference between the two signals $I_{1}(t)$ et $I_{2}(t)$. The operators differ by the definition of the adverb fuzzy set.

Figure 5(b) shows the application of the GreaterThan operator on the input signals shown in figures $1(\mathrm{a})$ and $1(\mathrm{~b})$ with the adverb "much" (figure 5(a)).

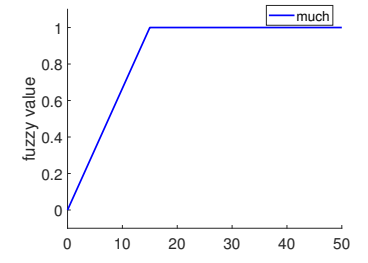

(a) Example of "much" membership function for a comparison with GreaterThan

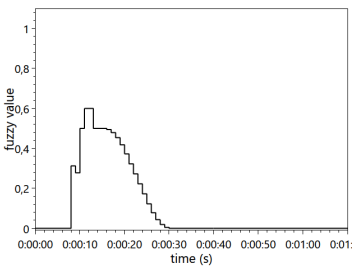

(b) "Input1 is much greater than input2 throughout the last 10 seconds"

Figure 5: Example of membership function for the adverb of the GreaterThan operator and result on Input 1 and Input 2

\section{Application to a drift detection}

The goal of the presented work is to apply fuzzy expert systems to predictive maintenance of complex systems. As illustration, we developed a specific software for wind turbines. Figures 6 show some screenshots of our tool. It provides an overview of the system (figure 6(a)) and can locate with a circle the suspected default. The tiles on the left indicate the state of each sub-system of the wind turbine : a green tile indicates it is fully functional while a red tile indicates a critical state. By clicking on a tile, it is possible to access a more detailed view (figure 6(b)) with the signals, the output of the fuzzy expert system, and the rules with their activation which give an explanation of the decision contrarily to other approaches.

In this paper, we focus on the characterization of one of the sub-systems: the rotor-side multicellular converter. It occasionally suffers from drifts which are clues that the energy production is not optimal. It consists of serial cells, each one containing two switches with complementary values. The combination of the values of all the switches in the converter defines a "mode". Among the 


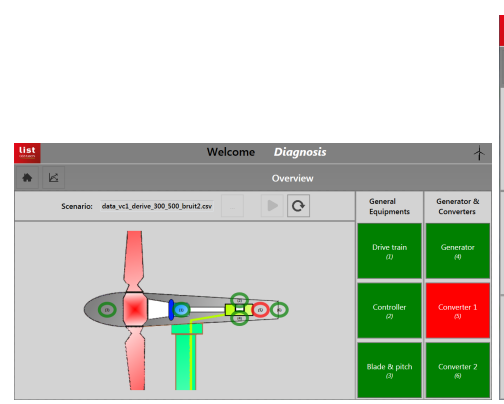

(a) Screenshot of the system overview

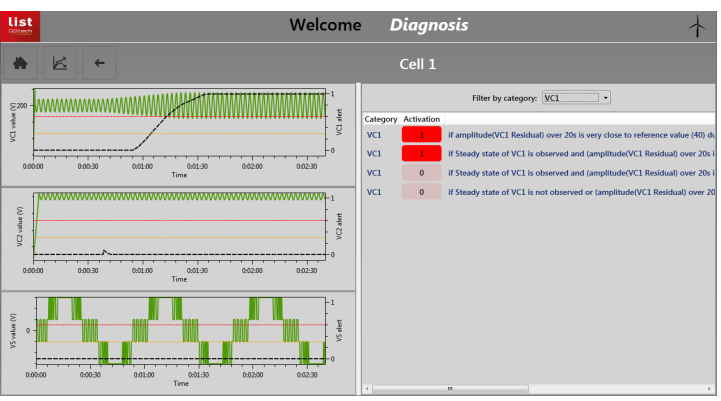

(b) Screenshot of the detailed view

Figure 6: Screenshots of the application for windturbines

other variables, the dynamics of the converter is also described by $V C_{i}$ which is the floating voltage of the capacitors $C_{i}$ of each cell. An instance of a controlled drift of $V C_{i}$ is shown in figure 7(a). According to the mode, the drift can be detected or not. It results in the computation of the new signal $V C_{i}$ residuals by subtracting the mean reference value to $V C_{i}$ according to the mode as defined in [13] (figure $7(\mathrm{~b})$ ). Then, we defined a rule base for detecting such a drift composed of:

- First, rules for defining the nominal values of the system. To compute that, we wait for a steady state during at least 20 seconds and we compare the actual values of the amplitude of $V C_{i}$ residuals to the values provided by the constructor.

- Then, rules for monitoring a drift and, according to its importance and its duration, to yield a suitable level of alarm.

Figures 8 show the membership functions used to compare the amplitude of $V C_{1}$ residuals (figure $7(\mathrm{c})$ ) to its reference value and different expressions computed to detect the drift. Once the steady state has been observed, the expression verifying that the amplitude of $V C_{i}$ residuals is very close to the reference value (figure $8(\mathrm{~b})$ ) is associated with a null alert (figure 9(a)), and the one verifying that it is much higher than the reference value (figure $8(\mathrm{c})$ ) is associated with a high alert. The defuzzified value of the alert is shown by a black curve in figure 9(b). The drift is applied between 40 and 80 seconds. It begins to be detected after only 15 seconds which is the delay necessary to compute the Ratio operator on the chosen temporal scope. Then, the alert value gradually rises until it reaches its maximum value 40 seconds afterward.

Monitoring a system with fuzzy temporal rules enables both to estimate a continuous value of the output (an alert here) and to know which rules are activated and led to the results. All the membership functions used as well as temporal scopes have to be chosen according to the application in order to characterize a normal or abnormal behavior of each sub-system. They can be learned when sufficient data of sub-systems are available. 


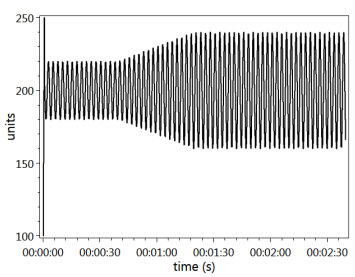

(a) $V C_{1}$ values

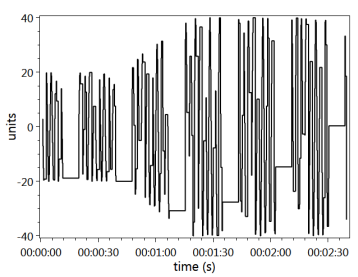

(b) $V C_{1}$ residuals

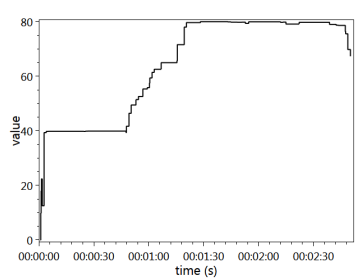

(c) Amplitude of $V C_{1}$ residuals over 20 seconds

Figure 7: Input example for floating voltage of capacitor $C_{1}$

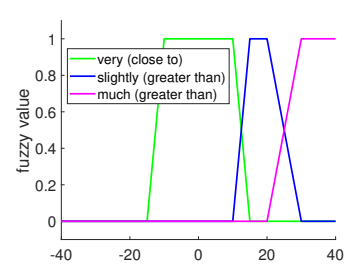

(a) Membership functions for comparing amplitudes of $V C_{1}$ residuals to the reference value

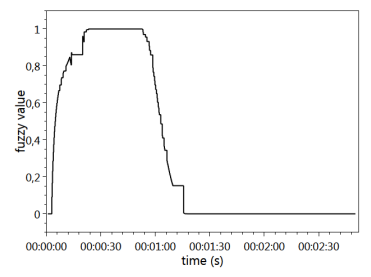

(b) "Amplitude of $V C_{1}$ residuals is very close to reference value during the last 20 seconds"

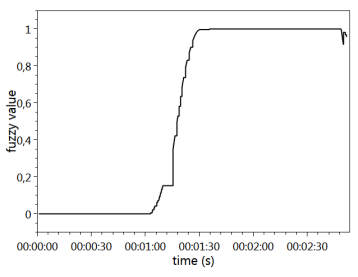

(c) "Amplitude of $V C_{1}$ residuals is much greater than the reference value during the last 20 seconds"

Figure 8: Examples of membership functions for comparing the amplitude of $V C_{1}$ residuals to the reference value and results of comparison operators

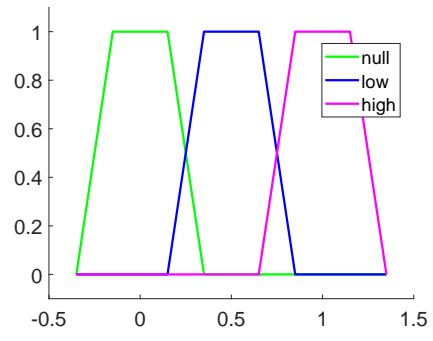

(a) Membership functions for output "alert"

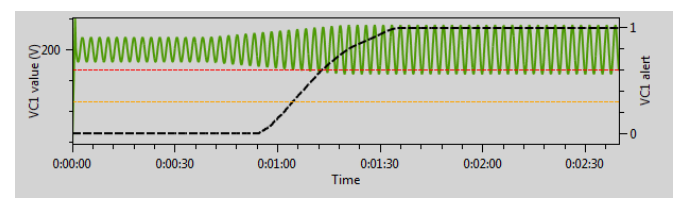

(b) $V C_{1}$ signal (left-hand axis) and defuzzified value of a drift alert between 0 and 1 (right-hand axis) superimposed

Figure 9: Example of membership functions for alerting a drift detection and application of detection rules on $V C_{1}$ signal

\section{Conclusion}

In this article, we use a compositional paradigm to build new temporal operators to characterize the kinetics of input values. From simple and intuitive 
operators like the ratio and the persistence, the temporal aspect is easily handled. These operators can take into account both the temporal uncertainty and the vagueness of the relation between the values.

With such operators, online fuzzy expert systems can play an important role in predictive maintenance or health monitoring. Experts can describe their knowledge about the systems and describe the clues which lead to damage detection from sensors signals. The decision making process can then be justified to the user by tracing activated rules. Moreover, such expert systems are independent of the system on which they are applied, contrary to statistical models which are system-dependent.

The perspectives of our work is to formalize more operators which are suitable for predictive maintenance, like online operators to characterize the seasonality or the periodicity of time-series.

\section{References}

[1] Barro, S., Bugarín, A., Cariñena, P., Díaz-Hermida, F., Mucientes, M.: Fuzzy temporal rule-based systems: New challenges. In: Actas del XIV Congreso Español sobre Tecnologías y Lógica Fuzzy (ESTYLF). pp. 507514. Langreo (Spain) (2008)

[2] Dubois, D., Hadj Ali, A., Prade, H.: Fuzziness and uncertainty in temporal reasoning. Journal of Universal Computer Science 9(9), 1168-1194 (2003)

[3] Hashemian, H.M., Bean, W.C.: State-of-the-art predictive maintenance techniques. IEEE Transactions on Instrumentation and Measurement 60(10), 3480-3492 (Oct 2011)

[4] Kobbacy, K.A.H.: Artificial Intelligence in Maintenance, pp. 209-231. Springer London, London (2008)

[5] Manaf, N.A.A., Beikzadeh, M.R.: Crisp-fuzzy representation of allen's temporal logic. In: Proceedings of the 25th Conference on Proceedings of the 25th IASTED International Multi-Conference: Artificial Intelligence and Applications. pp. 174-179. AIAP'07, ACTA Press, Anaheim, CA, USA (2007)

[6] Moyse, G., Lesot, M.J.: Linguistic summaries of locally periodic time series. Fuzzy Sets and Systems 285, 94 - 117 (2016), special Issue on Linguistic Description of Time Series

[7] Novák, V.: Linguistic characterization of time series. Fuzzy Sets and Systems 285, $52-72$ (2016), special Issue on Linguistic Description of Time Series

[8] Pereira, R.R., da Silva, V.A.D., Brito, J.N., Nolasco, J.D.: On-line monitoring induction motors by fuzzy logic: A study for predictive maintenance 
operators. In: 2016 12th International Conference on Natural Computation, Fuzzy Systems and Knowledge Discovery (ICNC-FSKD). pp. 13411346 (Aug 2016)

[9] Poli, J.P., Boudet, L., Mercier, D.: Online temporal reasoning for event and data streams processing. In: FUZZ-IEEE 2016. pp. 2257-2264 (July 2016)

[10] Schockaert, S., De Cock, M., Kerre, E.E.: Fuzzifying allen's temporal interval relations. Trans. Fuz Sys. 16(2), 517-533 (Apr 2008)

[11] Schockaert, S., Cock, M.D., Kerre, E.: Reasoning About Fuzzy Temporal and Spatial Information from the Web, Intelligent Information Systems, vol. 3. World Scientific (2010)

[12] da Silva Vicente, S.A., Fujimoto, R.Y., Padovese, L.R.: Rolling bearing fault diagnostic system using fuzzy logic. In: 10th IEEE International Conference on Fuzzy Systems. vol. 2, pp. 816-819 vol.3 (Dec 2001)

[13] Toubakh, H., Sayed-Mouchaweh, M.: Hybrid dynamic classifier for driftlike fault diagnosis in a class of hybrid dynamic systems: Application to wind turbine converters. Neurocomputing 171, 1496 - 1516 (2016) 